\title{
Alcohol and head and neck cancer risk in a prospective study
}

\author{
ND Freedman*, , A Schatzkin ${ }^{2}$, MF Leitzmann², AR Hollenbeck ${ }^{3}$ and CC Abnet ${ }^{2}$ \\ 'Cancer Prevention Fellowship Program, Division of Cancer Prevention, National Cancer Institute, National Institutes of Health, Executive Plaza North, \\ Suite 3109, 6130 Executive Boulevard, MSC 7361, Bethesda, MD 20892-7361, USA; ${ }^{2}$ Nutritional Epidemiology Branch, Division of Cancer \\ Epidemiology and Genetics, National Cancer Institute, NIH, 6120 Executive Blvd, EPS/320, MSC 7232, Rockville, MD 20852, USA; ${ }^{3}$ AARP, 601 E Street \\ NW, Washington, DC 20049, USA
}

\begin{abstract}
We investigated the relation between head and neck cancer risk and alcohol consumption in the NIH-AARP Diet and Health Study. During 2203500 person-years of follow-up, 6II men and 183 women developed head and neck cancer. With moderate drinking (up to one alcoholic drink per day) as the referent group, non-drinkers showed an increased risk of head and neck cancer (men: hazard ratio $(\mathrm{HR})$ 1.68, 95\% confidence interval (95\% Cl) 1.37-2.06; women: 1.46, 1.02-2.08). Among male and female alcohol drinkers, we observed a significant dose-response relationship between alcohol consumption and risk. The HR for consuming $>3$ drinks per day was significantly higher in women $(2.52,1.46-4.35)$ than in men $(1.48,1.15-1.90$; $P$ for interaction $=0.0036)$. The incidence rates per 100000 person-years for those who consumed $>3$ drinks per day were similar in men (77.6) and women (75.3). The higher HRs observed in women resulted from lower incidence rates in the referent group: women (I4.7), men (34.4). In summary, drinking $>3$ alcoholic beverages per day was associated with increased risk in men and women, but consumption of up to one drink per day may be associated with reduced risk relative to non-drinking.
\end{abstract}

British Journal of Cancer (2007) 96, I469-1474. doi: I0.1038/sj.bjc.66037| 3 www.bjcancer.com

Published online 27 March 2007

(c) 2007 Cancer Research UK

Keywords: head and neck cancer; alcohol; cohort

Each year, more than 600000 individuals are diagnosed with squamous cancers of the head and neck worldwide (Parkin et al, 2005). Head and neck cancer has multiple anatomic sub-sites, including the oral cavity, pharynx, and larynx, which may have distinct aetiologies.

Alcohol use has consistently been indicated as a risk factor for head and neck cancer (Sturgis et al, 2004). However, owing to small case numbers, previous prospective studies have typically collapsed cancers of the head and neck and the aesophagus into a single broad category of upper-gastrointestinal tract sites (Gronbaek et al, 1998; Kjaerheim et al, 1998; Boeing, 2002). Few prospective studies have examined the association between alcohol and individual head and neck cancer sub-sites, such as the larynx, oral cavity, or pharynx (Schottenfeld et al, 1974; Boffetta and Garfinkel, 1990; Adami et al, 1992; Tonnesen et al, 1994; Sigvardsson et al, 1996; Boffetta et al, 2001). Most of these studies have not adjusted for tobacco use, an important potential confounder of the relation between alcohol and head and neck cancer (Boffetta and Garfinkel, 1990). Also, few studies (Franceschi et al, 1994; Gronbaek et al, 1998; Hayes et al, 1999; Castellsague et al, 2004; Polesel et al, 2005) have examined non-drinkers and those that drink less than two drinks per day separately, an important comparison as consumption of less than two drinks per day may reduce cardiovascular disease risk (Maclure, 1993).

Although men are 2-7 times more likely to develop these cancers than women (depending on sub-site) (Parkin et al, 2005),

*Correspondence: Dr ND Freedman; E-mail: freedmanne@mail.nih.gov Received 29 January 2007; revised 26 February 2007; accepted 5 March 2007; published online 27 March 2007 few epidemiological studies have compared the association of alcohol and head and neck cancer in men to that in women (Blot et al, 1988; Franceschi et al, 1994; Hayes et al, 1999; Boffetta et al, 2001). These studies generally indicate higher relative risks in women than in men, although many had small case numbers and all but one were case-control studies precluding the examination of incidence rates.

Alcohol consumption is increasing in many countries worldwide, particularly among women (Boffetta and Hashibe, 2006), and this highlights the need to better understand the association between alcohol consumption and head and neck cancer. We prospectively investigated this association in 492960 participants of the NIH-AARP diet and health study.

\section{MATERIALS AND METHODS}

The establishment and recruitment procedures of the NIH-AARP Diet and Health Study have been described (Schatzkin et al, 2001). Between 1995 and 1996, a dietary questionnaire was mailed to 3.5 million members of AARP, formerly known as the American Association of Retired Persons, a large US organisation whose membership is open to those 50 years of age or older. These members resided in one of eight US states (California, Florida, Louisiana, New Jersey, North Carolina, Pennsylvania, Georgia, or Michigan). A total of 566407 respondents (308692 men and 211702 women) sufficiently completed the survey and consented to participate in the study. We excluded proxy respondents $(n=15760)$ and subjects with cancer at baseline $(n=51219)$, calorie intake more than two interquartile ranges from the mean $(n=4419)$, who died or were diagnosed with cancer on the first 
day of follow-up $(n=10)$, and who failed to provide information on alcohol use $(n=2039)$. The resulting analytical cohort comprised 492960 participants.

\section{Cohort follow-up}

Addresses for members of the NIH-AARP cohort were updated annually by matching the cohort database to that of the USA Postal Service change of address database (Michaud et al, 2005). Vital status was ascertained by annual linkage to the USA Social Security Administration death master file and responses to mailings. Follow-up time extended from study baseline (between 1995 and 1996) until diagnosis of a head and neck, aesophageal, or gastric cancer (as diagnosis of one of these cancers is associated with increased surveillance of the other sites), date of death, end of study (31 December 2000), or the date moved out of registry ascertainment area.

Incident cases of cancer were identified by probabilistic linkage between the NIH-AARP cohort membership and eight state cancer registry databases. We estimate that $90 \%$ of cancer cases are detected in the cohort by this approach (Michaud et al, 2005). Cancer sites were identified by anatomic site and histologic code of the International Classification of Disease for Oncology, third edition (Fritz, 2000). All cases of head and neck cancer with squamous histology were considered for this analysis. We classified tumours with site codes C32.0-C32.9 as laryngeal cancer. Oral cavity cancers included tumours with codes C00.1C06.9. Cancers of the oropharynx and hypopharynx included tumours of the tonsil (C09.0-C09.9), oropharynx (C10.0-C10.9), pyriform sinus (C12.9), hypopharynx (C13.0-C13.9), and pharynx not otherwise specified (NOS) (C14.0). The overarching head and neck cancer category included those diagnosed with a cancer of the oral cavity, oropharynx and hypopharynx, larynx, and those with other squamous tumours in the head or neck or overlapping region of the lip, oral cavity, and pharynx.

\section{Exposure assessment}

The baseline questionnaire contained questions about demographics, daily alcohol, cigarette use, physical activity, and a 124item food frequency questionnaire (Schatzkin et al, 2001). Usual daily alcohol (wine, beer, and liquor), fruit, and vegetable intakes were calculated from the questionnaire as pyramid servings as defined by the USA Department of Agriculture (USDA), taking account of intake frequency and serving size (Subar et al, 2000; Schatzkin et al, 2001). One drink corresponds to one serving of the USDA food guide pyramid: one 12 fluid ounce beer, one 5 fluid ounce glass of wine, or one 1.5 ounce shot of liquor (each approximately $13 \mathrm{~g}$ of alcohol). We used categorical variables of alcohol intake: none, up to 1 drink per day, 1-3 drinks per day, and $>3$ drinks per day. Self-administered questionnaires for alcohol intake have high reproducibility ( 0.84 after 4 years) and validity with respect to 1 week diet records ( 0.92 for men and 0.90 for women) (Giovannucci et al, 1991).

To adjust for smoking, we used smoking status (current, former, and never) and usual smoking dose to construct a categorical variable (smoke-quit-dose): never smokers, former smokers who smoked less than one pack per day, former smokers who smoked more than one pack per day, current smokers who smoked less than one pack per day, and current smokers who smoked more than one pack per day. We lacked information on smoking duration.

\section{Statistical analysis}

Analyses were performed with SAS version 8.2. Potential confounders were tabulated by alcohol intake. Hazard ratios (HR) and 95\% confidence intervals (95\% CI) were calculated using Cox proportional hazards regression (Cox, 1972). We tested the proportional hazards assumption by modelling interaction terms of time and categories of alcohol intake and found no statistically significant interactions. Follow-up time was used as the underlying time metric. Using age as the underlying time metric did not affect results. All multivariate models were adjusted by categorical variables for education, body mass index (World Health Organization categories), smoke-quit-dose, vigorous physical activity, activity throughout the day, and continuous measures for vegetable intake, fruit intake, total energy intake, and age at baseline. Owing to significant differences in the risk of nondrinkers relative to the risk of moderate alcohol drinkers of up to one drink per day, we used moderate drinkers as the referent category. The test for trend was restricted to alcohol drinkers and used a linear variable where each subject was assigned the median intake value for their category. Models that analysed categories of liquor, beer, or wine consumption further included the other two types of alcohol as categorical variables, to account for total alcohol consumption in each model. Small numbers of subjects had missing data values for some covariates. Missing covariates were represented by dummy variables in the models. Excluding those with missing covariate information slightly reduced case numbers but did not materially change point estimates. An alpha level of less than 0.05 was considered statistically significant and all tests were two sided. We tested for an interaction between categorical variables of alcohol intake and gender by using crossproduct terms with a three degree of freedom (df) likelihood ratio $\chi^{2}$ test. Age-adjusted incidence rates were calculated as in Breslow and Day (1987) with 5-year age bands and age- and gender-specific rates standardised to the entire NIH-AARP Diet and Health study population.

\section{RESULTS}

As shown in Table 1, alcohol drinkers were more likely to smoke and to have received more formal education than non-drinkers, but they consumed less fruit on average. The distribution of alcohol use within the AARP cohort, $24.1 \%$ (119007) non-drinkers, $53.1 \%$ (261 889) moderate drinkers of $0-1$ alcoholic beverages per day, $15.2 \%$ (74 854) drinkers of $1-3$ alcoholic beverages per day, and $7.5 \%$ (37210) drinkers of $>3$ drinks per day, was similar to that in the United States overall, as data from the 1999-2000 NHANES survey indicated $35.5 \%$ non-drinkers, $42.7 \%$ moderate drinkers of $0-1$ alcoholic beverages per day, $17.9 \%$ drinkers of $1-3$ alcoholic beverages per day, and $4.0 \%$ drinkers of $\geqslant 3$ alcoholic beverages per day (Breslow et al, 2006).

After 2203500 person-years of follow-up, 794 participants were diagnosed with head and neck cancer. We found that gender significantly modified the relation between alcohol and head and neck cancers $(P=0.0036,3 \mathrm{df})$ and present gender stratified estimates throughout. The age-standardised incidence rate per 100000 person-years among moderate drinking women (up to one drink per day) $(14.7,95 \%$ CI $11.3-18.0)$ was lower than moderate drinking (34.4, 30.0-39.0) men. Among drinkers, increasing alcohol consumption was significantly associated with increased risk. The incidence rates in those who drank $>3$ alcoholic drinks per day were similar in men and women (women: 75.3, 40.5-110.1; men: 77.6, 63.1-92.1) (Table 2). In age-adjusted models (Table 2), men and women who drank $>3$ alcoholic beverages per day were at increased risk relative to moderate drinkers (men: $2.25,1.79-$ 2.82; women: $5.16,3.08-8.63$ ). The association between increased alcohol consumption and risk among drinkers of alcohol was attenuated but remained statistically significant in multivariate adjusted models. Men (1.48, 1.15-1.90) and women $(2.52,1.46-$ 4.35) who drank $>3$ drinks per day were at increased risk relative to moderate drinkers (Table 3 ).

When examined by alcohol type, consuming more than three drinks of liquor or beer per day was associated with increased risk 
Table I Study characteristics by alcohol intake

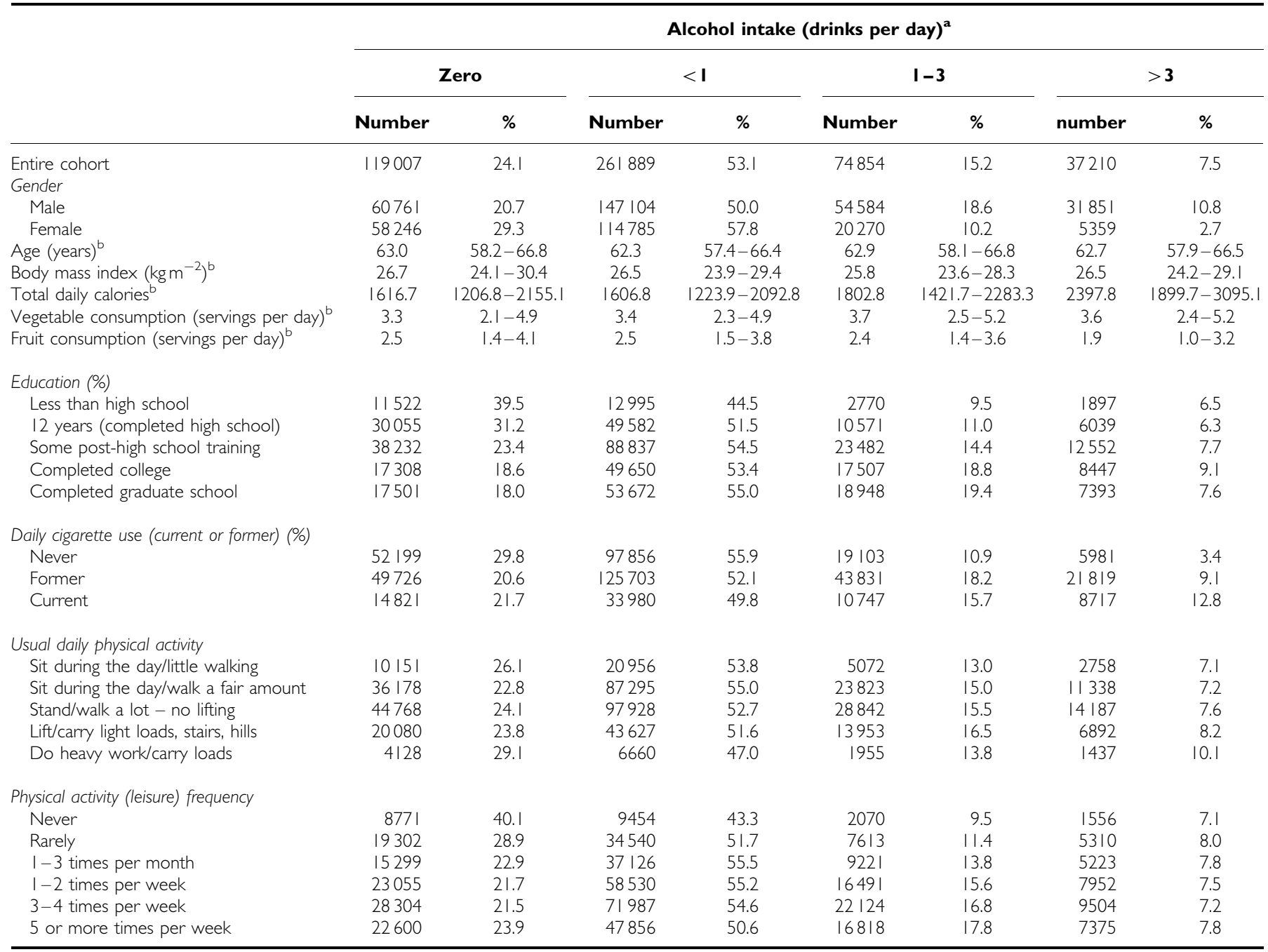

${ }^{a}$ Categories may not add up to 492960 persons owing to missing data. b Median (interquartile range).

Table 2 Age-adjusted risk ratios and incidence rates for head and neck cancer by alcohol use

\begin{tabular}{|c|c|c|c|c|c|}
\hline \multirow[b]{2}{*}{$\begin{array}{l}\text { Total alcohol } \\
\text { consumption }\end{array}$} & \multirow[b]{2}{*}{ Cases } & \multicolumn{4}{|c|}{ Age-adjusted } \\
\hline & & HR & $95 \% \mathrm{Cl}$ & $\begin{array}{c}\text { Incidence } \\
\text { rates }^{\mathbf{a}} \text { per } \\
100000 \\
\text { person- } \\
\text { years }\end{array}$ & $95 \% \mathrm{Cl}$ \\
\hline \multicolumn{6}{|l|}{ Men } \\
\hline 0 drinks per day & 165 & 1.77 & $1.45-2.16$ & 61.3 & $51.9-70.7$ \\
\hline$<$ I drink per day & 226 & 1.00 & & 34.4 & $30.0-39.0$ \\
\hline I-3 drinks per day & 110 & 1.29 & $1.03-2.82$ & 44.5 & $36.1-52.8$ \\
\hline$>3$ drinks per day & 110 & 2.25 & $1.79-2.82$ & 77.6 & $63.1-92.1$ \\
\hline \multicolumn{6}{|l|}{ Women } \\
\hline 0 drinks per day & 54 & 1.39 & $0.98-1.98$ & 20.4 & $14.9-25.8$ \\
\hline$<$ I drink per day & 75 & 1.00 & (ref) & 14.7 & $11.3-18.0$ \\
\hline - -3 drinks per day & 36 & 2.69 & $1.81-4.00$ & 39.6 & $26.6-52.5$ \\
\hline$>3$ drinks per day & 18 & 5.16 & $3.08-8.63$ & 75.3 & $40.5-110.1$ \\
\hline
\end{tabular}

$95 \% \mathrm{Cl}=95 \%$ confidence interval; $\mathrm{HR}=$ hazard ratio. ${ }^{\text {ancidence rates by alcohol }}$ category and gender were applied to the entire AARP population. relative to moderate consumption of these beverages in both men and women (Table 3).

We also investigated the association between alcohol intake and head and neck cancer sub-sites. Women who drank more than three alcoholic drinks per day were at significantly increased risk of oral cavity cancer $(2.81,1.29-6.11)$ and nonsignificantly increased risk of oropharyngeal and hypopharyngeal cancer $(1.97,0.42-9.31)$ and laryngeal cancer $(2.15,0.82-5.65)$ relative to moderate alcohol drinkers. Men who drank more than three alcoholic drinks per day were at significantly increased risk for cancers of the oral cavity (1.52, 1.01-2.27), oropharynx and hypopharynx $(2.32,1.29-4.18)$, but not the larynx compared to moderate drinkers (1.37, $0.91-2.05)$ (Table 4).

In both age-adjusted models (Table 2; men: 1.77, 1.45-2.16; women: $1.39,0.98-1.98)$ and multivariate adjusted models (Table 3; men: 1.68, 1.37-2.06; women: 1.46, 1.02-2.08), nondrinkers were at increased risk of head and neck cancer relative to moderate alcohol drinkers. When examined by alcohol type (Table 3), non-drinking men were at increased risk relative to moderate drinkers of wine $(1.34,1.10-1.63)$, beer $(1.18,0.96$ $1.45)$, and liquor $(1.28,1.03-1.58)$, whereas non-drinking women were at increased risk relative to moderate drinkers of wine (1.58, $1.12-2.25)$ and liquor $(1.16,0.80-1.66)$. 
Table 3 Adjusted $\mathrm{HR}$ and $95 \% \mathrm{Cl}$ for alcohol intake and head and neck cancer

\begin{tabular}{|c|c|c|c|c|c|c|}
\hline & \multicolumn{6}{|c|}{ Head and neck cancer ${ }^{a}$} \\
\hline & \multicolumn{3}{|c|}{ Men } & \multicolumn{3}{|c|}{ Women } \\
\hline & Cases & HR & $95 \% \mathrm{Cl}$ & Cases & HR & $95 \% \mathrm{Cl}$ \\
\hline \multicolumn{7}{|l|}{ Total alcohol } \\
\hline 0 drinks per day & 165 & 1.68 & $1.37-2.06$ & 54 & 1.46 & $1.02-2.08$ \\
\hline$<$ I drink per day & 226 & 1.00 & (ref) & 75 & 1.00 & (ref) \\
\hline I-3 drinks per day & 110 & 1.23 & $0.98-1.55$ & 36 & 1.99 & $1.33-2.99$ \\
\hline$>3$ drinks per day & 110 & 1.48 & $1.15-1.90$ & 18 & 2.52 & $1.46-4.35$ \\
\hline$P$-value for trend ${ }^{b}$ & & 0.001 & & & 0.0002 & \\
\hline \multicolumn{7}{|l|}{ Wine $^{c}$} \\
\hline 0 drinks per day & 318 & 1.34 & $1.10-1.63$ & 91 & 1.58 & $1.12-2.25$ \\
\hline$<$ I drink per day & 252 & 1.00 & (ref) & 74 & 1.00 & (ref) \\
\hline I-3 drinks per day & 40 & 1.16 & $0.83-1.62$ & 18 & 1.97 & $|.17-3.3|$ \\
\hline$>3$ drinks per day & I & 0.43 & $0.06-3.05$ & 0 & & \\
\hline$P$-value for trend ${ }^{b}$ & & 0.872 & & & 0.332 & \\
\hline \multicolumn{7}{|l|}{ Beer $^{d}$} \\
\hline 0 drinks per day & 230 & 1.18 & $0.96-1.45$ & 107 & 0.85 & $0.60-1.19$ \\
\hline$<$ I drink per day & 286 & 1.00 & (ref) & 67 & 1.00 & (ref) \\
\hline I-3 drinks per day & 41 & 1.24 & $0.89-1.72$ & 3 & 0.85 & $0.27-2.73$ \\
\hline$>3$ drinks per day & 54 & $|.6|$ & $1.16-2.22$ & 6 & 3.40 & $1.40-8.24$ \\
\hline$P$-value for trend ${ }^{b^{\prime}}$ & & 0.002 & & & 0.084 & \\
\hline \multicolumn{7}{|l|}{ Liquor $^{\mathrm{e}}$} \\
\hline 0 drinks per day & 294 & 1.28 & $1.03-1.58$ & 92 & 1.16 & $0.80-1.66$ \\
\hline$<$ I drink per day & 195 & 1.00 & (ref) & 62 & 1.00 & (ref) \\
\hline I-3 drinks per day & 61 & 1.53 & $1.14-2.04$ & 17 & 1.86 & $1.08-3.19$ \\
\hline$>3$ drinks per day & 61 & 1.85 & $1.37-2.50$ & 12 & 2.25 & $1.19-4.26$ \\
\hline$P$-value for trend ${ }^{b^{\prime}}$ & & $<.0001$ & & & 0.003 & \\
\hline
\end{tabular}

95\% Cl=95\% confidence interval; HR = hazard ratio. ${ }^{a}$ Adjusted for gender, age at entry into cohort, body mass index, education, smoke-quit-dose, vigorous physical activity, usual activity throughout the day, fruit intake, vegetable intake, and total energy. ${ }^{b}$ The test for trend was restricted to alcohol drinkers and used a linear variable where each subject was assigned the median intake value for their category. cAdditionally adjusted for categories of beer and liquor. ${ }^{\mathrm{d}}$ Additionally adjusted for categories of liquor and wine. ${ }^{e}$ Additionally adjusted for categories of wine and beer.

We performed several sensitivity analyses. Years of education and alcohol intake were positively correlated (Pearson correlation of 0.12 ); so we stratified our analysis on years of education. The stratified estimates for non-drinkers relative to moderate drinkers were $1.70(1.21-2.37)$ for men and $2.57(1.20-5.53)$ for women who had a college education or greater and $1.76(1.35-2.27)$ for men and $1.35(0.89-2.03)$ for women without a college education. We also stratified by smoking status (current, former, and never). Non-drinking men and women were at elevated risk relative to moderate drinkers among current (men: 2.00, 1.39-2.88; women: $1.28,0.77-2.13$ ), former (men: $1.58,1.17-2.13$; women: $1.77,0.94-$ 3.34 ), and never smokers (men: $1.35,0.85-2.15$; women: 1.53 , $0.59-3.94)$. We also excluded those who reported poor health on the questionnaire. After excluding 34580 men and 25013 women who reported poor health at baseline, non-drinking men (1.73, $1.32-2.17)$ and non-drinking women $(1.67,1.12-2.51)$ were at increased risk relative to moderate drinkers. Similarly, after excluding the first 2 years of follow-up, non-drinking participants remained at increased risk (men: 1.59, 1.21-2.09; women: 1.35, $0.94-4.25)$.

\section{DISCUSSION}

In a large prospective study, we investigated the association between alcohol and head and neck cancer in men and women separately. Consistent with previous studies (Blot et al, 1988; Boffetta and Garfinkel, 1990; Adami et al, 1992; Franceschi et al, 1994; Gronbaek et al, 1998; Kjaerheim et al, 1998; Hayes et al, 1999; Boffetta et al, 2001; Boeing, 2002; Castellsague et al, 2004; Sturgis et al, 2004; Polesel et al, 2005; Boffetta and Hashibe, 2006), predominantly of men, we observed a significant dose-response association between increasing alcohol consumption and head and neck cancer in both male and female alcohol drinkers. Results were generally similar for beer, liquor, and wine, although only $0.3 \%$ (1528) of the cohort (1064 men and 464 women) drank $>3$ glasses of wine per day limiting power. Hazard ratios for head and neck cancer sub-sites were also generally similar.

In this study, although the absolute age-standardised incidence rates among those who drank 1-3 alcoholic drinks per day and those who drank $>3$ alcoholic drinks per day were similar in men and women, the HR for the association between alcohol intake and head and neck cancer risk were significantly higher for women than for men. Similar increased risks among women were observed in most previous studies of this association (Blot et al, 1988; Franceschi et al, 1994; Hayes et al, 1999; Boffetta et al, 2001). In our study, the higher HR observed in women compared to men result from lower incidence rates in the referent group. These differences in baseline incidence suggest the presence of additional gender-specific risk or protective factors. These factors might include occupational carcinogens (Boffetta et al, 2003) or reproductive hormones (Yang et al, 1989; Olsson et al, 2003).

Compared to not-drinking, drinking up to one alcoholic drink per day was associated with reduced head and neck cancer risk. Although some previous epidemiological studies have observed a protective association between moderate alcohol consumption and cancers of the head and neck and the aesophagus, results overall have been inconsistent (Franceschi et al, 1994; Gronbaek et al, 1998; Hayes et al, 1999; Castellsague et al, 2004; Polesel et al, 2005). Most previous studies were conducted in populations that drink larger amounts of alcohol (Altieri et al, 2005) than that of the current study.

The observed inverse association could reflect exposure misclassification if former heavy drinkers had stopped drinking at the time of the questionnaire. Excluding either those subjects who reported poor health or the first 2 years of follow-up did not appreciably alter risk estimates and alcohol consumption was assessed before cancer diagnosis. However, some studies suggest that former drinkers remain at elevated risk for head and neck cancer, even 10 years after use (Hayes et al, 1999; Castellsague et al, 2004). Because we lacked information on past alcohol use, misclassification of non-drinkers may have affected our risk estimates.

It is also possible that moderate alcohol drinking is a proxy for a healthy or privileged lifestyle or an associated exposure. We did adjust models for head and neck cancer risk factors as well as markers of lifestyle, including age, education, body mass index, smoking, physical activity, fruit intake, vegetable intake, and total energy intake. However, the observed protective association could still be due to an unmeasured or poorly measured confounder in our analysis, such as socioeconomic status (SES). Years of education, as with the other covariates used for adjustment, did not attenuate the protective association. We additionally explored SES as a confounder using median income of the census tract in which the participant lived. Inclusion of this variable in the multivariate analysis did not alter risk estimates (data shown). We also examined possible effect modification by smoking status, as health conscious individuals may be more likely to drink moderate quantities of alcohol and less likely to smoke. Although the risk estimates for moderate alcohol consumption were similar among current, former, and never smokers, few cases with head and neck cancer were non-smokers ( 95 men and 20 women), limiting the power to investigate this stratum. 
Adjusted $\mathrm{HR}$ and $95 \% \mathrm{Cl}$ for head and neck cancer subtypes by gender

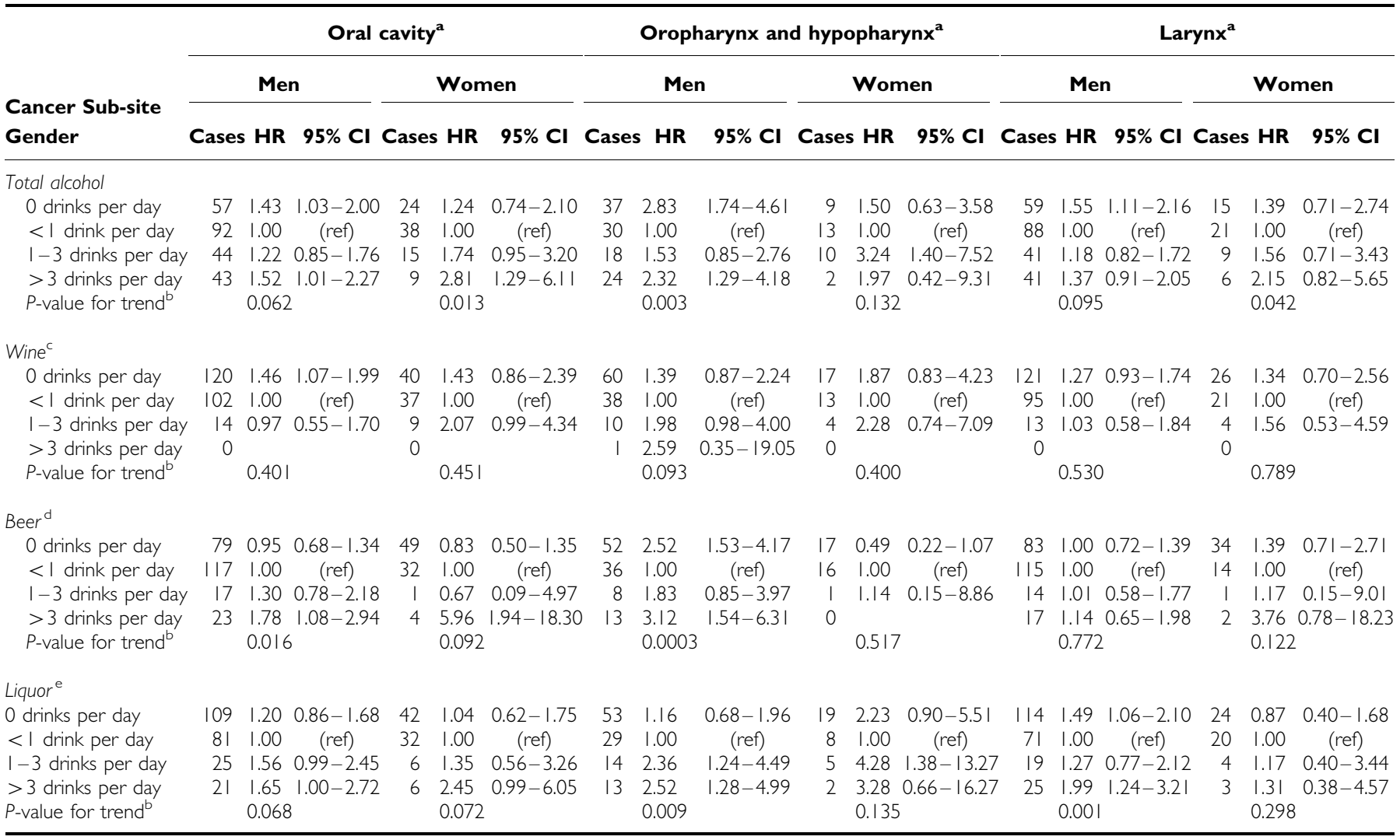

$95 \% \mathrm{Cl}=95 \%$ confidence interval; $\mathrm{HR}=$ hazard ratio. ${ }^{a}$ Adjusted for gender, age at entry into cohort, body mass index, education, smoke-quit-dose, vigorous physical activity, activity throughout the day, fruit intake, vegetable intake, and total energy. ${ }^{\mathrm{b}}$ The test for trend was restricted to alcohol drinkers and used a linear variable where each subject was assigned the median intake value for their category. ${ }^{~}$ Additionally adjusted for categories of beer and liquor. ${ }^{\mathrm{d}}$ Additionally adjusted for categories of liquor and wine. ${ }^{\mathrm{e}}$ Additionally adjusted for categories of wine and beer.

This study has a number of strengths. It is a large prospective analysis with exposure information collected before cancer diagnosis. We present models separately for men and women and we examined the associations for both total head and neck cancer and by sub-sites. To limit confounding, we adjusted our models for most of the major risk factors for head and neck cancer, including cigarette smoking, a strong risk factor that is associated with alcohol use. Furthermore, the large number of participants who consumed less than one alcoholic drink per day allowed us to investigate the association between moderate drinking and risk. This study also had several limitations. We lacked information on participant income and occupation, factors that could confound the association between alcohol intake and risk. We also lacked information about past alcohol consumption and smoking duration.

In conclusion, among alcohol drinkers, increasing consumption of alcohol was associated with increased risk of head and neck cancer in both men and women. Although consumption of $>3$ drinks per day had a bigger HR for head and neck cancer in women than in men, incidence rates for men and women in this category were similar. Men and women who reported consuming less than one drink per day had lower rates of head and neck cancer than men and women who reported no alcohol consumption. The observed association between increasing alcohol consumption and increased head and neck cancer in men and women is consistent with many previous studies. In contrast, the observed protective association with drinking up to one alcoholic beverage per day should be interpreted cautiously and must be investigated further, particularly in prospective studies that collect information on lifetime use of alcohol.

\section{ACKNOWLEDGEMENTS}

This research was supported by the Intramural Research Program of the NIH, National Cancer Institute. Cancer incidence data from the Atlanta metropolitan area were collected by the Georgia Center for Cancer Statistics, Department of Epidemiology, Rollins School of Public Health, Emory University. Cancer incidence data from California were collected by the California Department of Health Services, Cancer Surveillance Section. Cancer incidence data from the Detroit metropolitan area were collected by the Michigan Cancer Surveillance Program, Community Health Administration, State of Michigan. The Florida cancer incidence data used in this report were collected by the Florida Cancer Data System under contract to the Department of Health (DOH). The views expressed herein are solely those of the authors and do not necessarily reflect those of the contractor or DOH. Cancer incidence data from Louisiana were collected by the Louisiana Tumor Registry, Louisiana State University Medical Center in New Orleans. Cancer incidence data from New Jersey were collected by the New Jersey State Cancer Registry, Cancer Epidemiology Services, New Jersey State Department of Health and Senior Services. 


\section{REFERENCES}

Adami HO, McLaughlin JK, Hsing AW, Wolk A, Ekbom A, Holmberg L, Persson I (1992) Alcoholism and cancer risk: a population-based cohort study. Cancer Causes Control 3: 419-425

Altieri A, Garavello W, Bosetti C, Gallus S, La Vecchia C (2005) Alcohol consumption and risk of laryngeal cancer. Oral Oncol 41: 956-965

Blot WJ, McLaughlin JK, Winn DM, Austin DF, Greenberg RS, PrestonMartin S, Bernstein L, Schoenberg JB, Stemhagen A, Fraumeni Jr JF (1988) Smoking and drinking in relation to oral and pharyngeal cancer. Cancer Res 48: 3282 - 3287

Boeing $H$ (2002) Alcohol and risk of cancer of the upper gastrointestinal tract: first analysis of the EPIC data. IARC Sci Publ 156: $151-154$

Boffetta P, Garfinkel L (1990) Alcohol drinking and mortality among men enrolled in an American Cancer Society prospective study. Epidemiology 1: $342-348$

Boffetta P, Hashibe M (2006) Alcohol and cancer. Lancet Oncol 7: 149-156

Boffetta P, Richiardi L, Berrino F, Esteve J, Pisani P, Crosignani P, Raymond L, Zubiri L, Del MA, Lehmann W, Donato F, Terracini B, Tuyns A, Merletti F (2003) Occupation and larynx and hypopharynx cancer: an international case-control study in France, Italy, Spain, and Switzerland. Cancer Causes Control 14: 203-212

Boffetta P, Ye W, Adami HO, Mucci LA, Nyren O (2001) Risk of cancers of the lung, head and neck in patients hospitalized for alcoholism in Sweden. Br J Cancer 85: 678-682

Breslow NE, Day NE (1987) Statistical Methods in Cancer Research. Lyon: International Agency for Research on Cancer

Breslow RA, Guenther PM, Smothers BA (2006) Alcohol drinking patterns and diet quality: the 1999-2000 National Health and Nutrition Examination Survey. Am J Epidemiol 163: 359-366

Castellsague X, Quintana MJ, Martinez MC, Nieto A, Sanchez MJ, Juan A, Monner A, Carrera M, Agudo A, Quer M, Munoz N, Herrero R, Franceschi S, Bosch FX (2004) The role of type of tobacco and type of alcoholic beverage in oral carcinogenesis. Int J Cancer 108: 741 - 749

Cox DR (1972) Regression Models and Life-Tables. J R Stat Soc Ser B Stat Methodol 34: 187

Franceschi S, Bidoli E, Negri E, Barbone F, La Vecchia C. (1994) Alcohol and cancers of the upper aerodigestive tract in men and women. Cancer Epidemiol Biomarkers Prev 3: 299-304

Fritz AG (2000) International Classification of Diseases for Oncology : ICD-O. Geneva: World Health Organization

Giovannucci E, Colditz G, Stampfer MJ, Rimm EB, Litin L, Sampson L, Willett WC (1991) The assessment of alcohol consumption by a simple self-administered questionnaire. Am J Epidemiol 133: 810-817

Gronbaek M, Becker U, Johansen D, Tonnesen H, Jensen G, Sorensen TI (1998) Population based cohort study of the association between alcohol intake and cancer of the upper digestive tract. BMJ 317: 844-847
Hayes RB, Bravo-Otero E, Kleinman DV, Brown LM, Fraumeni Jr JF, Harty LC, Winn DM (1999) Tobacco and alcohol use and oral cancer in Puerto Rico. Cancer Causes Control 10: $27-33$

Kjaerheim K, Gaard M, Andersen A (1998) The role of alcohol, tobacco, and dietary factors in upper aerogastric tract cancers: a prospective study of 10,900 Norwegian men. Cancer Causes Control 9: 99-108

Maclure M (1993) Demonstration of deductive meta-analysis: ethanol intake and risk of myocardial infarction. Epidemiol Rev 15: $328-351$

Michaud DS, Midthune D, Hermansen S, Leitzmann M, Harlan LC, Kipnis V, Schatzkin A (2005) Comparison of cancer registry case ascertainment with SEER estimates and self-reporting in a subset of the NIH-AARP Diet and Health Study. J Registry Manage 32: 70-75

Olsson H, Bladstrom A, Ingvar C (2003) Are smoking-associated cancers prevented or postponed in women using hormone replacement therapy? Obstet Gynecol 102: $565-570$

Parkin DM, Bray F, Ferlay J, Pisani P (2005) Global cancer statistics, 2002. CA Cancer J Clin 55: 74-108

Polesel J, Dal ML, Bagnardi V, Zucchetto A, Zambon A, Levi F, La Vecchia C., Franceschi S (2005) Estimating dose-response relationship between ethanol and risk of cancer using regression spline models. Int $J$ Cancer 114: $836-841$

Schatzkin A, Subar AF, Thompson FE, Harlan LC, Tangrea J, Hollenbeck AR, Hurwitz PE, Coyle L, Schussler N, Michaud DS, Freedman LS, Brown CC, Midthune D, Kipnis V (2001) Design and serendipity in establishing a large cohort with wide dietary intake distributions: the National Institutes of Health-American Association of Retired Persons Diet and Health Study. Am J Epidemiol 154: 1119-1125

Schottenfeld D, Gantt RC, Wyner EL (1974) The role of alcohol and tobacco in multiple primary cancers of the upper digestive system, larynx and lung: a prospective study. Prev Med 3: 277-293

Sigvardsson S, Hardell L, Przybeck TR, Cloninger R (1996) Increased cancer risk among Swedish female alcoholics. Epidemiology 7: $140-143$

Sturgis EM, Wei Q, Spitz MR (2004) Descriptive epidemiology and risk factors for head and neck cancer. Semin Oncol 31: 726-733

Subar AF, Midthune D, Kulldorff M, Brown CC, Thompson FE, Kipnis V, Schatzkin A (2000) Evaluation of alternative approaches to assign nutrient values to food groups in food frequency questionnaires. $A m \mathrm{~J}$ Epidemiol 152: 279-286

Tonnesen H, Moller H, Andersen JR, Jensen E, Juel K (1994) Cancer morbidity in alcohol abusers. Br J Cancer 69: 327-332

Yang PC, Thomas DB, Daling JR, Davis S (1989) Differences in the sex ratio of laryngeal cancer incidence rates by anatomic subsite. J Clin Epidemiol 42: $755-758$ 